\title{
Extrauterine pregnancies-risk factors and management ${ }^{*}$
}

\author{
Anna Patapuro, Henri Lahdemaki, Jaana Marttala, Markku Santala, Markku Ryynanen ${ }^{\#}$ \\ Department of Obstetrics \& Gynecology, Oulu University Hospital, Oulu, Finland \\ Email: ${ }^{\#}$ markku.ryynanen@,oulu.fi \\ Received 20 February 2013; revised 25 March 2013; accepted 3 April 2013 \\ Copyright (C) 2013 Anna Patapuro et al. This is an open access article distributed under the Creative Commons Attribution License, \\ which permits unrestricted use, distribution, and reproduction in any medium, provided the original work is properly cited.
}

\begin{abstract}
Objective: To study the clinical progress, risk factors, management, and outcomes of suspected ectopic pregnancy (EP). Design: Retrospective clinical case study. Population: All 184 patients hospitalized for suspected EP during the period 1.1.2008-31.12.2011. Setting: Oulu University Hospital, Finland. Methods: The clinical progress, risk factors, management, complications and outcomes were based on studies of hospital records. Main outcome measures: Symptoms, serum quantitative human chorionic gonadotropin (hCG) levels, management, complications and outcome of patients. Results: At least one of the risk factors was found in 117 women (66\%). There were 11 patients without symptoms. In the initial visit, the median hCG concentration was 1915 (20 - 73,000) IU/l. The most common treatment was surgical, 137/181 (76\%), followed with medical treatment, 22/181 (12\%) and expectant management, 22/181 (12\%). Conclusions: Surgery was the most widely used treatment for EP. We might thus draw attention to more conservative management.
\end{abstract}

Keywords: Ectopic Pregnancy; Methotrexate; Salpingec Tomy; Salpingostomy; Expectant; Management; Risk Factor

\section{INTRODUCTION}

In ectopic pregnancy (EP), the gestational sac is outside the normal uterus. Approximately $95 \%$ of EPs are located in the Fallopian tube [1]. EPs may also occur in the cervix or in intra-abdominal structures like the ovary or a cesarean scar. EPs cause a significant morbidity in women of reproductive age and even, rarely, maternal death $[2,3]$. Of all reported pregnancies in developing countries, $1 \%-2 \%$ are EPs. In Finland, the incidence rate

\footnotetext{
${ }^{*}$ The authors have no conflicts of interest to report.

No specific funding.

${ }^{\#}$ Corresponding author.
}

was $1.5 \%$ in 2010 [4]. The incidence in the United States increased from 4.5 to 19.7 per 1000 reported pregnancies between 1972 and 1992, possibly due to advanced diagnostics and a higher prevalence of risk factors such as smoking and pelvic inflammatory disease (PID) $[5,6]$.

A combination of symptoms (bleeding, pelvic pain etc.), transvaginal ultrasonography (tubal ring, fluid in Pouch of Douglas, etc.) and repeated measurement of serum human chorionic gonadotropin (hCG) concentrations still constitute the suspicion of EP $[1,3]$. Many potential serum biomarkers that could simplify the diagnosis of EP are under research (e.g. inhibin A and vascular endothelial growth factor (VEGF)) [7]. Early diagnosis is important in allowing the use of all treatment options and the retention of optimal fertility $[1,8]$. EP may be managed expectantly, medically or surgically. The choice of treatment depends on a given woman's hemodynamic condition, presenting symptoms, location, gestation week, and wish for future fertility.

EP is still a crucial issue. The aim of this study is to evaluate the clinical progress, risk factors, management, complications and outcomes of suspected EP.

\section{MATERIAL AND METHODS}

The study group comprised 184 consecutive patients hospitalized for suspected EP during the study period between January 1, 2008 and December 31, 2011. The follow-up time was from nine months to 4.5 years. We collected all the information (age, gestational week, risk factors, symptoms, hCG concentrations, type of management, complications and outcome) from hospital records. We regarded as risk factors cigarette smoking, previous pelvic and abdominal surgery, infertility and assisted reproductive technology (ART), previous EP, previous salpingectomy, intrauterine contraceptive device and confirmed genital infection or pelvic inflammatory disease, including Chlamydia trachomatis. We have drawn attention to the exact location and have focused on the management and outcomes of EP. 


\section{RESULTS}

In seven of 184 women $(3.8 \%)$ the EP could not be confirmed. In one case there was intrauterine pregnancy and two cases ended in miscarriage. In addition, in four cases we could not localize the pregnancy, so there were in fact 177 cases with confirmed EP. The median age of the patients was 29 (range 17 - 49) year and parity 1.0 (range 0 - 9). The median for ongoing gestational week was 6.0 (range 2 - 11). At least one of the risk factors was found in $117(66 \%)$ women (Table 1). There were 11 patients without any symptoms. EP was suspected while visiting the doctor in early pregnancy $(\mathrm{N}=7)$ or in a follow-up visit after ART $(\mathrm{N}=2)$ and after previous EP $(\mathrm{N}=2)$. At admission to hospital $170(94 \%)$ patients had symptoms, mostly vaginal bleeding or/and abdominal pain. Of those women with symptoms 119 (66\%) had acute abdominal pain. The median initial hCG level was $1915(20$ - 73,000) IU/1. The hCG level increased in 41 (23\%) and decreased in 62 (34\%) women during the follow-up. In 78 women the hCG change was unknown, because these patients underwent surgery soon after admission to hospital. The incidence of heterotopic pregnancy in our material was $1.7 \%$ (3/181). In five cases (2.8\%) EP developed after post coital hormonal contraception. There were 80 (44\%) women who became pregnant subsequent to EP.

In most cases, 162 (90\%) women, EP was located in the Fallopian tube (Table 2). The pregnancy was located in the cornual region $(\mathrm{N}=7)$, in the cervix $(\mathrm{N}=4)$, in pregnancy of unknown location (PUL) $(\mathrm{N}=4)$, in the ovary $(\mathrm{N}=3)$ and in the cesarean scar $(\mathrm{N}=1)$. In tubal pregnancies the exact location was not mentioned in records for 66 women. In the rest of the cases EP was located in 64 patients in the ampulla, and in 5 women proximally. In 27 cases there was a sign in laparoscopy of tubal abortion. Of all tubal pregnancies (include cornual pregnancies) $57 \%(97 / 169)$ were located on the right side and $42 \%(71 / 169)$ on the left side and $0.6 \%(\mathrm{~N}$ $=1$ ) bilaterally.

\subsection{Expectant Management}

Expectant management was attempted in 32 (17\%) women (Table 3). The success rate for expectant management was $69 \%(22 / 32)$. In this group there were no significant difference in serum hCG level in women with successful management, median hCG 486 (20 - 2767) IU/1, compared to those with failed management, median 408 (80 - 5269) IU/1. In the expectant management group, the initial serum hCG concentration was under $1000 \mathrm{IU} / 1$ in 26 women. In this group hCG levels were decreasing in $84 \%(27 / 32)$ and increasing in $9.4 \%$ (3/32) of women. All three women in the increasing hCG-level group needed further treatment. We did not have information of
Table 1. The incidence of risk factors for ectopic pregnancy in women treated for ectopic pregnancy in Oulu University Hospital 2008-2011.

\begin{tabular}{|c|c|c|}
\hline Risk factors for ectopic pregnancy & $\mathbf{N}$ & $\%$ \\
\hline No risk factors & 65 & 36 \\
\hline Previous spontaneous or induced abortion & 63 & 35 \\
\hline At least one previous pelvic surgery & 62 & 34 \\
\hline Cesarean section & 20 & 11 \\
\hline Salpingectomy & 18 & 9.9 \\
\hline Laparoscopy & 15 & 8.3 \\
\hline Appendectomy & 14 & 7.7 \\
\hline Laparotomy & 5 & 2.8 \\
\hline Infertility problems & 44 & 24 \\
\hline Age $>35$ years & 43 & 24 \\
\hline ART & 29 & 16 \\
\hline IVF & 11 & 6.1 \\
\hline Insemination & 6 & 3.3 \\
\hline Medical treatment & 4 & 2.2 \\
\hline Previous ART & 8 & 4.4 \\
\hline Previous EP & 22 & 12 \\
\hline Previous chlamydia and PID & 22 & 12 \\
\hline Chlamydia trachomatis & 14 & 7.7 \\
\hline PID & 8 & 4.4 \\
\hline Smoking & 16 & 8.8 \\
\hline Intrauterine contraceptive device & 1 & 0.6 \\
\hline All & 181 & 100 \\
\hline
\end{tabular}

ART: Assisted reproductive technology; IVF: in vitro fertilization; EP: ectopic pregnancy; PID: pelvic inflammatory disease.

level change in two cases. In the failed expectant treatment group nine pregnancies were located in tube (in ampulla $\mathrm{N}=3$, exact location was not mentioned $\mathrm{N}=6$ ) and one in PUL. After failed expectant management $60 \%$ $(6 / 10)$ of patients managed with methotrexate and $40 \%$ (4/10) with salpingectomy.

\subsection{Medical Treatment}

As medical treatment we used systemic methotrexate (only one case was treated with mifepristone and misoprostol). The median serum hCG concentration in women treated with methotrexate was $589 \mathrm{IU} / 1$ (78 - 72,630 IU/1). In total 29 women were managed medically, and 
Table 2. Crosstabulation of location and treatments of ectopic pregnancies in Oulu University Hospital 2008-2011.

\begin{tabular}{|c|c|c|c|c|c|c|c|}
\hline \multirow{2}{*}{ Treatment } & \multicolumn{6}{|c|}{ Location of EP } & \multirow{2}{*}{ Tota } \\
\hline & Fallopian tube & Cesarean scar & Ovary & Cervix & Cornu & PUL & \\
\hline Expectant management & 17 & 0 & 0 & 1 & 0 & 3 & 21 \\
\hline Methotrexate single dose & 11 & 0 & 0 & 3 & 1 & 1 & 16 \\
\hline Methotrexate 2 doses & 1 & 1 & 0 & 0 & 0 & 0 & 2 \\
\hline Methotrexate 3 doses & 0 & 0 & 0 & 0 & 2 & 0 & 2 \\
\hline Salpingectomy & 121 & 0 & 0 & 0 & 1 & 0 & 122 \\
\hline Salpingostomy & 9 & 0 & 0 & 0 & 0 & 0 & 9 \\
\hline Salpingostomy and methotrexate & 1 & 0 & 0 & 0 & 0 & 0 & 1 \\
\hline Laparatomy & 1 & 0 & 0 & 0 & 1 & 0 & 2 \\
\hline Laparoscopy & 1 & 0 & 3 & 0 & 0 & 0 & 4 \\
\hline Methotrexate 10 doses & 0 & 0 & 0 & 0 & 1 & 0 & 1 \\
\hline Mifepristone and misoprostol & 0 & 0 & 0 & 0 & 1 & 0 & 1 \\
\hline Total & 162 & 1 & 3 & 4 & 7 & 4 & 181 \\
\hline
\end{tabular}

EP: ectopic pregnancy; PUL: pregnancy of unknown location

Table 3. Management success rates and used further management.

\begin{tabular}{ccccc}
\hline Management & N & Succesful & Failed & Further management \\
\hline Expectant & 32 & 22 & 10 & Methotrexate N $=6$ \\
Medical & 29 & 22 & 7 & Surgery N $=4$ \\
Surgery & 137 & & & Surgery N $=7$ \\
Salpingostomy & 12 & 10 & 2 & \\
Salpingectomy & 122 & 122 & 0 & Methotrexate N $=1$ \\
Laparotomy & 2 & 2 & 0 & \\
Laparoscopy & 4 & 181 & 20 & 1 \\
Total & 201 & & & \\
\hline
\end{tabular}

22 of them successfully (76\%). In $73 \%$ (16/22) of cases patients were managed with single dose methotrexate and $23 \%(5 / 22)$ with multiple-dose methotrexate. One cornual pregnancy needed 10 methotrexate doses. In seven cases medical treatment failed and all women were treated with salpingectomy. Furthermore, one woman needed a single dose of methotrexate after salpingostomy.

\subsection{Surgery}

Surgery $(\mathrm{N}=137)$ was the most common management for EP. The median serum hCG concentration in women treated surgically was $2566 \mathrm{IU} / 1$ (209 - 73,000 IU/1). Salpingostomy was used in 12 patients, but two of them needed further management. In 122 cases (67\%) salpingectomy was performed with a success rate of nearly $100 \%$. One cornual pregnancy was treated with laparotomy and one salpingectomy was also made via laparotomy. All EPs located in the ovary $(\mathrm{N}=3)$ were managed via laparoscopy. One failure occurred in cornual pregnancy that needed to be treated with three methotrexate doses after laparoscopy. Altogether, $7.3 \%$ of patients $(10 / 137)$ received conservative surgery, and radical surgery was used in $93 \%$ of women (128/137).

\subsection{Complications}

Bleeding during surgical operation was recorded in only $65 \%(89 / 138)$ of operations' notes. The median amount of bleeding was $600 \mathrm{ml}$ (range 0 - 5000) including both old and new leaks. In addition, diagnostic laparoscopy had been performed for $6(3.3 \%)$ of 181 women. These pregnancies were tubal abortion $(\mathrm{N}=2)$, intratubal pregnancy $(\mathrm{N}=1)$, cornual pregnancy $(\mathrm{N}=2)$ and one PUL. There were $22(12 \%)$ women who sought medical attention after treatment. These women had at least one of the complications introduced in Table 4.

\section{DISCUSSION}

In reality the number of patients suspected must be greater since we collected only those cases with definitive diagnosis. In hospital records it is impossible to dis- 
Table 4. Of all women $12 \%$ had at least one complication after EUP treatment.

\begin{tabular}{|c|c|c|c|c|c|}
\hline Complication & Salpingectomy & Methotrexate & Expectant & Laparoscopy & Laparotomy \\
\hline Abdominal pain & 10 & 3 & 1 & 1 & \\
\hline Vaginal bleeding & 5 & 1 & 1 & & \\
\hline Hematoma & 2 & 1 & & & \\
\hline Fever & 3 & & & & \\
\hline Endometritis & 1 & & 1 & & \\
\hline Vaginitis & 1 & & & & \\
\hline Skeletal anomaly & & & & & 1 \\
\hline Nausea & 1 & & & & \\
\hline Pyelonephritis & & & & & 1 \\
\hline Appendicitis & 1 & & & & \\
\hline Incision leak & 1 & & & & \\
\hline Total & 25 & 5 & 3 & 1 & 2 \\
\hline
\end{tabular}

cover those patients with suspicion of EP who do not seek medical care.

\subsection{Expectant Management}

Some EPs can recover spontaneously. Expectant management means a conservative procedure that can be safe and effective for appropriately selected women [9]. Expectant management can be offered to asymptomatic, clinically stable women with small adnexal masses $(<4$ $\mathrm{cm})$, low s-hCG concentration $(<1000 \mathrm{IU} / 1)$ and declining serum hCG level [10]. In this study $12 \%(22 / 181)$ of EP were managed expectantly. About $25 \%-40 \%$ of tubal pregnancies may be managed expectantly [11-13] but in our material only $11 \%(18 / 162)$ of tubal pregnancies were managed expectantly. Success rates vary from $47 \%$ to $82 \%$ depending on the women's initial status [3,12]. In our study the success rate for expectant management was $69 \%(22 / 32)$. Expectant management is especially recommended for PULs $[15,16]$.

\subsection{Medical Treatment}

The most common medical treatment is methotrexate [17]. Methotrexate is very effective against EP because it interferes with DNA synthesis and cell multiplication, thus preventing the proliferation of trophoblastic tissue $[3,18]$. Methotrexate $\left(50 \mathrm{mg} / \mathrm{m}^{2}\right.$ or $\left.1 \mathrm{mg} / \mathrm{kg}\right)$ can be given systematically (i.m.) or locally and as a single dose or in multiple doses $[2,3,19]$. Methotrexate treatment is useful for women who are hemodynamically stable and have light symptoms with low serum hCG-level (<5000 IU/1), small size of EUP $(\leq 4 \mathrm{~cm})$, unruptured tubal pregnancy and no signs of active bleeding or cardiac motion. Full blood count and liver and renal function tests should be done before management. In the large study (11,989 EPs) that estimated treatment of EP in the United States from
2002 to 2007 methotrexate treatment increased from $11 \%$ in 2002 to $35 \%$ in 2007 (p < 0.001) [20]. Unfortunately, expectantly managed EPs were not included in that study. In our study, 12\% (22/181) of EPs were treated medically. The methotrexate failure rate in Hoover's study [20] was $15 \%$ and in our data $25 \%(7 / 28)$. Methotrexate management seems to be more cost effective than laparoscopy $[8,18,19]$, so this increase in methotrexate usage can offer savings for health care systems. The overall success rate for methotrexate treatment is $89 \%$ [21], but in this data it was 75\% (21/28). About $36 \%$ of women experienced side effects that are typically minor. In this data $14 \%(3 / 22)$ of women treated with methotrexate experienced side effects. The most common side effects are mild stomatitis and gastrointestinal upset such as abdominal pain. Methotrexate is used for both tubal and interstitial pregnancies [1].

\subsection{Surgical Management}

The oldest and still the most effective treatment for EPs is surgery $[1,3]$. Laparoscopic treatment decreases postoperative pain, intraoperative blood loss, costs, hospital stay and speeds up the return to normal life compared with laparotomy. Laparoscopy is a standard method, especially in women who are hemodynamically stable. In tubal pregnancies, the surgical approach is either salpingostomy or salpingectomy. A salpingostomy is the removal of EP without removal of the tube, whereas salpingectomy is the removal of the affected tube. If a patient's contralateral tuba is abnormal and if she desires future fertility, then a laparoscopic salpingostomy should be performed. When future fertility is not desired, the contralateral tuba is normal or the affected tube is damaged, then salpingectomy is the preferred management. After conservative salpingostomy, the risk of persistent trophoblast is approximately $8 \%$ and, furthermore, the 
risk of tubal bleeding is increased [1,2]. In our study, $17 \%(2 / 12)$ of patients experienced persistent trophoblast after salpingostomy. The risk of subsequent EP is $15 \%$ after salpingostomy and only $10 \%$ after salpingectomy. For these reasons, salpingostomy should be used only for women who wish to have another pregnancy. Surgery still seems to be the most used treatment for EP. In this data $76 \%(137 / 181)$ of women were managed surgically. In one study, however, surgical treatment with laparotomy decreased between 2002 and 2007 from $40 \%$ to $33 \%(p<0.001)[20]$. In that study, both laparoscopy and methotrexate treatments were substituted for laparotomy. In our material, EPs were treated mainly laparoscopically, allowing for a less invasive procedure, whereas laparotomy was used for only $1.5 \%(2 / 137)$ of women. Side effects after surgery occurred in $12 \%$ of women. It may be the case that we manage EPs surgically far too often.

Approximately $95 \%$ of EPs are tubal pregnancies [1]. In our study $11 \%(19 / 181)$ of pregnancies were located extratubally. It is worth mentioning here that we included PULs in our study (4/181). These PULs can also sometimes be spontaneous abortions. Pregnancy in the cesarean scar is a rare form of EP, but its rate is increasing worldwide [22]. Of all EPs with a history of at least one cesarean delivery $6.1 \%$ were located in the cesarean scar [23]. We had one cesarean scar pregnancy in a woman with a history of three cesarean deliveries. In our data $5.0 \%(1 / 20)$ of women with a history of cesarean section had pregnancy in the cesarean scar.

The incidence of spontaneous heterotopic pregnancy is about 1:30,000 and after ART it is 1:100 [24]. In our data the frequency of heterotopic pregnancy was very high at 3 out of 181 EPs. One was a spontaneous pregnancy and two of them started after ART. Although emergency contraceptive pills are not associated with an increased risk of EP [25], 2.8\% (5/181) of our patients reported the use of emergency contraceptive pills.

In this retrospective study, we gathered information from hospital records. It is possible that there appears to be a lack of some useful information resulting from recorded anamnesis. In our data, only $65 \%(89 / 138)$ of operation reports included details of bleeding. Clinicians should pay close attention to writing up epicrisis thoroughly.

\section{REFERENCES}

[1] Ehrenberg-Buchner, S., Sandadi, S., Moawad, N., Pinkerton, J. and Hurd, W. (2009) Ectopic pregnancy: Role of laparoscopic treatment. Clinical Obstetrics and Gynecology, 52, 372-379. doi:10.1097/GRF.0b013e3181b0be24

[2] Murray, H., Baakdah, H., Bardell, T. and Tulandi, T. (2005) Diagnosis and treatment of ectopic pregnancy. Canadian Medical Association, 173, 905-912.

\section{doi:10.1503/cmaj.050222}

[3] Sivalingam, V.N., Duncan, W.C., Kirk, E., Shephard, L.A. and Horne, A.W. (2011) Diagnosis and management of ectopic pregnancy. Journal of Family Planning \& Reproductive Health Care, 37, 231-240. doi:10.1136/jfprhc-2011-0073

[4] National Institute for Health and Welfare (2012).

[5] Goldner, T.E., Lawson, H.W., Xia, Z. and Atrash, H,K. (1993) Surveillance for ectopic pregnancy-United States, 1970-1989. MMWR CDC Surveillance Summary, 42, 7385.

[6] Centers for Disease Control and Prevention (CDC) (1995) Ectopic pregnancy-United States, 1990-1992. MMWR Morbidity and Mortality Weekly Report, 44, 46-48.

[7] Rausch, M.E., Sammel, M., Takacs, P., Chung, K., Shaunik, A. and Barnhart, K.T. (2011) Development of a multiple marker test for ectopic pregnancy. Obstetrics \& Gynecology, 117, 573-582. doi:10.1097/AOG.0b013e31820b3c61

[8] Mol, F., Mol, B.W., Ankum, W.M., van der Veen, F. and Hajenius, P.J. (2008) Current evidence on surgery, systemic methotrexate and expectant management in the treatment of tubal ectopic pregnancy: A systematic review and meta-analysis. Human Reproduction Update, 14, 309-319. doi:10.1093/humupd/dmn012

[9] Kirk, E. and Bourne, T. (2006) The nonsurgical management of ectopic pregnancy. Current Opinion in $\mathrm{Ob}$ stetrics and Gynecology, 18, 587-593. doi:10.1097/GCO.0b013e3280105886

[10] Ramakrishnan, K. and Scheid, D.C. (2006) Ectopic pregnancy: Expectant management or immediate surgery? An algorithm to improve outcomes. The Journal of Family Practice, 55, 517-522.

[11] Makinen, J.I., Kivijarvi, A.K. and Irjala K.M. (1990) Success of non-surgical management of ectopic pregnancy. Lancet, 335, 1099. doi:10.1016/0140-6736(90)92674-7

[12] Korhonen, J., Stenman, U.H. and Ylostalo, P. (1994) Serum human chorionic gonadotropin dynamics during spontaneous resolution of ectopic pregnancy. Fertility and Sterility, 61, 632-636.

[13] Elson, J., Tailor, A., Banerjee, S., Salim, R., Hillaby, K. and Jurkovic, D. (2004) Expectant management of tubal ectopic pregnancy: Prediction of successful outcome using tree analysis. Ultrasound in Obstetrics and Gynecology, 23, 552-556. doi:10.1002/uog.1061

[14] Shalev, E., Peleg, D., Tsabari, A., Romano, S. and Bustan, M. (1995) Spontaneous resolution of ectopic pregnancy: Natural history. Fertility and Sterility, 63, 15-19.

[15] Condous, G., Okaro, E. and Bourne, T. (2005) Pregnancies of unknown location: Diagnostic dilemmas and management. Current Opinion in Obstetrics and Gynecology, 17, 568-573. doi:10.1097/01.gco.0000191515.07019.cb

[16] Stevens, E. and Gilbert-Cohen, J. (2007) Surgical considerations in early pregnancy: Ectopic pregnancy and ovarion torsion. The Journal of Perinatal \& Neonatal Nursing, 21, 22-29.

[17] Hajenius, P.J., Mol, F., Mol, B.W., Bossuyt, P.M.M., 
Ankum, W.M. and Van der Veen, F. (2009) Interventions for tubal ectopic pregnancy. The Cochrane Database of Systematic Reviews, 24.

[18] Miller, J.H. and Griffin, E. (2003) Methotrexate administration for ectopic pregnancy in the emergency department-One hospital's protocol/competencies. Journal of Emergency Nursing, 29, 240-244.

[19] Buster, J.E. and Heard, M.J. (2000) Current issues in medical management of ectopic pregnancy. Current Opinion in Obstetrics and Gynecology, 12, 525-527. doi:10.1097/00001703-200012000-00012

[20] Hoover, K., Tao, G. and Kent, C. (2010) Trends in the diagnosis and treatment of ectopic pregnancy in the United States. Obstetrics and Gynecology, 115, 495-502. doi:10.1097/AOG.0b013e3181d0c328

[21] Barnhart, K.T., Gosman, G., Ashby, R. and Sammel, M. (2003) The medical management of ectopic pregnancy: A meta-analysis comparing "single dose" and "multidose" regimens. Obstetrics and Gynecology, 101, 778-784. doi:10.1016/S0029-7844(02)03158-7

[22] Sowter, M.C. and Farquhar, C.M. (2004) Ectopic pregnancy: An update. Current Opinion in Obstetrics and Gynecology, 16, 289-293. doi:10.1097/01.gco.0000136494.49334.6b

[23] Rotas, M., Haberman, S. and Levgur, M. (2006) Caesarean scar ectopic pregnancies: Etiology, diagnosis, and management. Obstetrics and Gynecology, 107, 13731381. doi:10.1097/01.AOG.0000218690.24494.ce

[24] Lemus, J.F. (2000) Ectopic pregnancy: An update. Current Opinion in Obstetrics and Gynecology, 12, 369-375. doi:10.1097/00001703-200010000-00005

[25] Cleland, K., Raymond, E., Trussell, J., Cheng, L. and Zhu, H. (2010) Ectopic pregnancy and emergency contraceptive pills: A systematic review. Obstetrics and Gynecology, 115, 1263-1266. doi:10.1097/AOG.0b013e3181dd22ef

\section{ABBREVIATION}

ART: assisted reproductive technology;

EP: ectopic pregnancy;

hCG: human chorionic gonadotropin;
PID: pelvic inflammatory disease;

PUL: pregnancy of unknown location;

VEGF: vascular endothelial growth factor. 